\title{
What is there to fear from something so small?
}

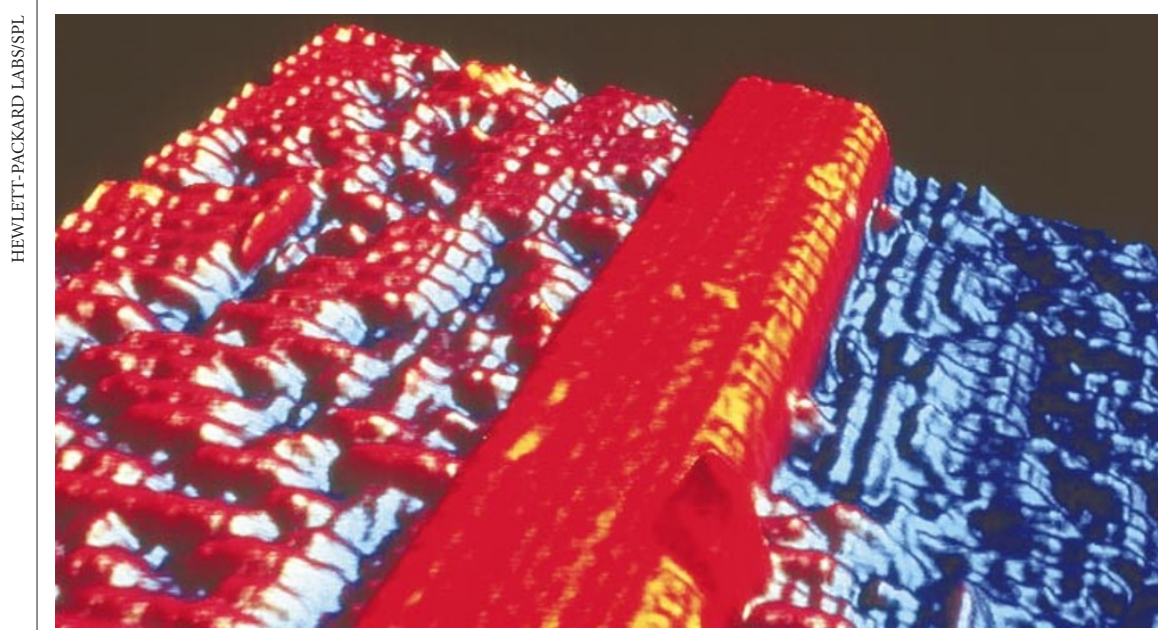

In miniature: nanowires such this one, just 10 atoms wide, are seen by many as the future of electronics.

$\mathrm{N}$ ext March, Mark Welland's laboratory at the University of Cambridge, UK, will gain an unusual member of staff. Welland's team works on nanometredimension wires and tubes that could form the future of electronics, but the new recruit won't be an engineer or a physicist - he or she will be a social scientist.

The appointment - a two-year position that will include running regular meetings with everyone from industry representatives to green activists - acknowledges public fears about the possible effects of nanotechnology on human health and the environment. Although Welland may not subscribe to long-standing scare stories about a 'grey goo' of nanometre-sized robots taking over the planet, he realizes that scientists need to address this and other concerns head on.

Welland's proactive attitude stems in large part from a desire to avoid a rerun of Europe's experience with genetically modified (GM) crops. Over the past decade, vociferous campaigning by environmental groups, combined with an inability of both biotech firms and scientists to understand and respond to public concerns, has created a hostile climate of public opinion. Although the discussion has — in Britain, at least — matured this year into a more detailed scientific consideration of the environmental risks and benefits of individual GM crops, these negative perceptions are now deeply ingrained.

Given this background, Welland and other nanotechnologists are alarmed by signs that their work will be the next target for environmental campaigners. Media attention began to build after the publication in January of The Big Down, a report by the ETC group, a small environmental organization based in Winnipeg, Canada. In its previous guise as the Rural Advancement Foundation
International, ETC had a history of influential campaigning against GM agriculture.

The ETC report argued that synthetic nanoparticles could be toxic. The Big Down and other ETC publications went on to envisage a technology out of control, potentially able to create new weapons of mass destruction or novel life forms.

ETC doesn't subscribe to the old 'grey goo' scenario. But it has cleverly married fears about GM with its campaign against nanotech by arguing that the public should be especially concerned about 'nanobiotechnology'. This future industry, ETC claims, could create a 'green goo' of novel organisms and products that will behave in unpredictable and uncontrollable ways

Ask ETC officials to define what, exactly, they mean by nanobiotechnology and they become frustratingly difficult to pin down — when pushed, they mention experiments in which researchers have altered the bases of DNA to create a novel, larger molecule that can still store genetic information (H. Liu et al. Science 302, 868-871; 2003). Such fundamental projects are easy to portray as playing God with the stuff of life. But in reality, they have little to do with creating an industry based on new life forms. And even if such work did eventually find applications, most scientists are confident that they could be regulated effectively.

Despite the currently nebulous nature of the 'green goo' scare, the idea may still have resonance with the public. Nanotechnologists would be unwise to dismiss ETC's campaign - particularly as many toxicologists agree that too little is known about the effect on human health of breathing in nanoparticles.

In Britain, the scientific establishment has been relatively quick to respond. In June, the Royal Society and the Royal Academy of
Engineering launched a review of the social and ethical implications of nanotechnology, which should report next spring. In the United States, a bill passed in November recommending some $\$ 4$ billion in spending on nanotech over the next four years also calls for a federal advisory panel to look into similar issues. But federal officials remain concerned that US nanotechnologists are poorly equipped to engage in a debate on the risks and benefits posed by their work. "Because of the GM debate, the United Kingdom is ahead of us in developing mechanisms for dialogue," says Julia Moore, an expert on nanotechnology policy at the National Science Foundation in Arlington, Virginia.

It's hard to say whether efforts to address the concerns raised by the ETC group will help to avoid the polarization of views that has afflicted the GM debate in Europe. But if scientists don't get involved, debate about nanotechnology will simply be conducted without them. "I've learnt from the GM debate," says Welland. "It's easy to condemn a technology, but hard to fight back." Jim Giles

\section{Genomics}

\section{Compare and contrast}

Nore, more, more! That is the cry from comparative geneticists, who can't wait to get their hands on newly minted genome sequences. Thanks to the growing availability of such data, their research has gathered unprecedented momentum over the past twelve months. They have gained fresh insights into the regulatory sequences that control the activity of each organism's genes, and into the evolutionary forces that have shaped modern genomes.

"Before, it was like a cottage industry comparing bits of sequence here and therebut now we're routinely comparing whole genomes with implications that are much farther reaching," says Evan Eichler, a comparative geneticist at Case Western Reserve University in Cleveland, Ohio.

Tracing the 'footprints' of regulatory sequences involves comparing the genomes of several related species. Make multiple comparisons, and conserved regulatory elements should stand out from the 'noise' of sequences shared between close relatives simply through common descent. The more genomes the better, according to researchers who this year completed such comparisons for several related yeast species ${ }^{1,2}$. 


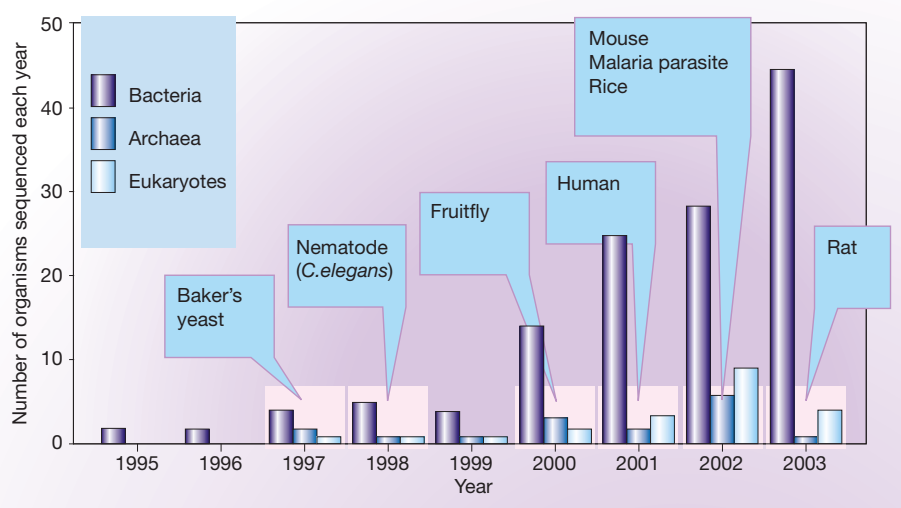

Comparative success: the rising number of sequenced genomes is bringing evolutionary insights.

Analyses of this type are particularly informative if they include both near and far evolutionary relatives - as was illustrated by reports that identified potential regulatory elements in the human genome by comparing chunks of our sequence with those of vertebrates as diverse as other primates, the platypus, chickens and fish ${ }^{3-5}$.

Comparisons between species have also revised estimates of the total number of genes possessed by important lab organisms. The tally for the baker's yeast Saccharomyces cerevisiae, for instance, has been reduced by 500 genes, following the analysis of three related yeast species ${ }^{1}$. Meanwhile, the nematode Caenorhabditis elegans has gained 1,300 or so genes from comparisons with its cousin C. briggsae ${ }^{6}$. Our own gene tally, as recorded on the Ensembl genome-browser website, has slipped this year from about 31,000 to just under 25,000, following comparisons with various other vertebrate sequences.

Comparative analysis has also taught us a thing or two about genome evolution. Over the eons, genomes have been flipped and flopped, added to and chopped, to give rise to new genes and entire gene families. Through cross-species comparisons, it is possible to determine when these changes arose. Comparing various bits of human sequences with those of other primates ${ }^{4,7}$, for instance, has revealed events that have sculpted our genome throughout evolution. The Y chromosome $^{8}$, for example, seems to have a neat trick for copying important male-specific genes to protect them from being lost as the chromosome degrades down the generations.

Researchers now want to devise experiments to explore the predictions made by comparative genomics. For instance, Mark Johnston of the Washington University School of Medicine in St Louis, Missouri, is putting the proposed regulatory elements identified in yeast ${ }^{2}$ to the test in various ways. One assay involves throwing them onto 'chips' carrying proteins known to bind to regulatory regions of DNA, to see if any of them take the bait.

Carina Dennis
1. Kellis, M., Patterson, N., Endrizzi, M., Birren, B. \& Lander, E. S. Nature 423, 241-254 (2003).

2. Cliften, P. et al. Science 301, 71-76 (2003).

3. Boffelli, D. et al. Science 299, 1391-1394 (2003).

4. Thomas, J. W. et al. Nature 424, 788-793 (2003).

5. Dermitzakis, E. T. et al. Science 302, 1033-1035 (2003).

6. Stein, L. D. et al. PLoS Biol. 1, 166-192 (2003).

7. Frazer, K. A. et al. Genome Res. 13, 341-346 (2003).

8. Rozen, S. et al. Nature 423, 873-876 (2003)

www.ensembl.org

\section{Cosmology}

\section{Welcome to the real world}

t has often been easy for cynics to dismiss cosmologists as whimsical purveyors of 'just-so' stories. But 2003 will go down as the year in which cosmology became a precise observational science - and for that we can thank NASA's Wilkinson Microwave Anisotropy Probe (WMAP), a satellite launched in 2001 that has been studying the faint afterglow of the Big Bang.

In February, WMAP produced an all-sky map, with unprecedented resolution, of the ancient microwaves that fill the Universe. By combining WMAP data with measurements from ground-based microwave telescopes, researchers have pinned down the Universe's age to a relatively sprightly 13.7 billion years, give or take about 200 million. They have also verified that the cosmos is overwhelmingly made up of two shadowy constituents: dark matter and dark energy. Dark matter, invisible mass whose gravitational pull helps to shape galaxies, seems to make up about a quarter of the Universe, whereas dark energy, which accounts for almost everything else, is a mysterious phenomenon that pushes matter apart at the largest scales. That leaves only about $4 \%$ for the visible stuff such as stars, planets and clouds of interstellar gas ${ }^{1}$.

WMAP's microwave picture agreed with other results obtained this year, such as measurements of distant, exploding stars" . "The degree of cosmic consistency is really heartening," says Charles Bennett, lead scientist on the WMAP project at NASA's Goddard Space Flight Center in Greenbelt, Maryland.

But the data did contain a few surprises. Most notably, fluctuations in the temperature and polarization of the microwaves detected by WMAP showed that the first generation of stars and galaxies appeared just 200 million years after the Big Bang ${ }^{1}$ hundreds of millions of years earlier than most theorists had thought.

Measurements of microwaves from disparate points of the sky also present something of a mystery, according to John Carlstrom, an astronomer at the University of Chicago. The temperature differences between these points match poorly with standard theories, he says. This has led to some new ideas about the shape of the Universe: it could, for example, be dodecahedral, rather like a soccer ball ${ }^{3}$.

That will give the theorists some thinking to do, as their favourite hypothesis has been that the Universe is flat and infinite. Happily, the shape of the Universe can be probed by future data on the microwave background from WMAP, ground-based telescopes and the European Space Agency's Planck satellite, which will carry instruments of even greater precision and is set to launch in 2007.

\section{Geoff Brumfiel}

1. Bennett, C. L. et al. Astrophys. J. Suppl. 148, 1-27 (2003)

2. Riess, A. G. et al. Preprint at $<$ http://www.arxiv.org/abs/ astro-ph/0308185> (2003)

3. Lumiet, J.-P. et al. Nature 425, 593-595 (2003).

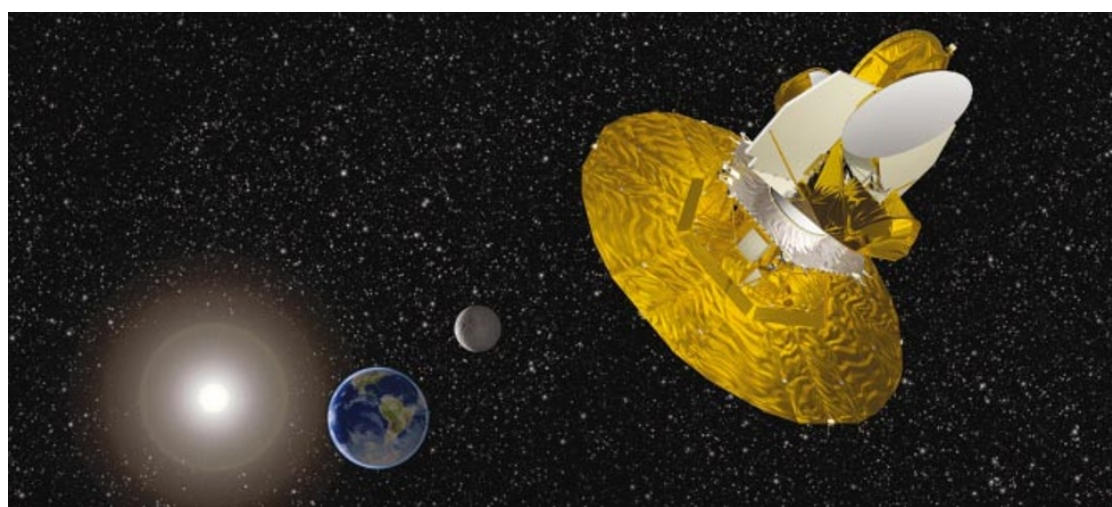

Glowing report: the WMAP spacecraft has helped to quantify the composition of the Universe. 